\title{
First evaluation of organotellurium derivatives as carbonic anhydrase I, II, IV, VII and IX inhibitors
}

\author{
Andrea Angeli, ${ }^{a}$ Damiano Tanini, ${ }^{\text {b Antonella Capperucci, }}{ }^{\text {b Claudiu T. Supuran }}{ }^{\text {** }}$ \\ ${ }^{a}$ Department of University of Florence, NEUROFARBA Dept., Sezione di Scienze Farmaceutiche, Via Ugo Schiff 6, 50019 Sesto \\ Fiorentino (Florence), Italy \\ ${ }^{b}$ Department of University of Florence, Department of Chemistry "Ugo Schiff", Via della Lastruccia 13, I-50019 Sesto Fiorentino, Italy.
}

\begin{abstract}
A series of tellurides was evaluated as carbonic anhydrase (CA, EC 4.2.1.1) inhibitors against the human (h) carbonic anhydrase isoforms hCA I, II, IV, VII and IX, involved in a variety of diseases, including glaucoma, retinitis pigmentosa, epilepsy, arthritis and tumors. These compounds, which are the first tellurium-containing derivatives acting as inhibitors of carbonic anhydrase enzymes, showed effective inhibition against all isoforms investigated and some of them were selective for inhibiting the cytosolic or the membrane-bound CAs. Thus, these carbonic anhydrase inhibitors are interesting leads for the development of isoform-selective inhibitors.
\end{abstract}

Keywords: carbonic anhydrase; inhibitor, metalloenzymes, tellurium, telluride

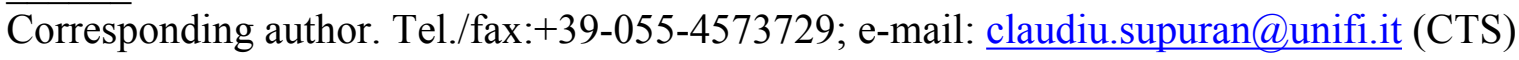




\section{Introduction}

Tellurium is a rare element and, unlike the other group VI members (e.g., $\mathrm{O}, \mathrm{S}$, and Se), which have many biological applications, this element has not any known such function. ${ }^{1}$ During the last decades, inorganic tellurium derivatives were employed for the development of innovative materials, such as fluorescent CdTe quantum dots as probes in biological detection, ${ }^{2,3}$ telluride clusters, nanoparticles and nanotubes, which found potential applications in the electronics and medicine fields. ${ }^{4-6}$ At the same time, a range of organotellurium compounds were developed, being shown that they possess a range of unique properties such as interesting activity against pathogenic microorganisms, ${ }^{7,8}$ inhibition of cancer cells growth, ${ }^{9-11}$ potent caspase and cathepsin inhibitory propieties ${ }^{12}$ and antioxidant activity, which are often superior to those of the selenium analogues. ${ }^{13,14}$ In this particular context, we should mention carbonic anhydrase (CAs, EC 4.2.1.1) an enzyme that plays an important role in many physiological and pathological processes associated with $\mathrm{pH}$ control, ion transport, fluid secretion and biosynthetic reactions. ${ }^{19-21}$ Recent studies from our group ${ }^{15}$ reported that some of the many CA isoforms known to date, more precisely, the human (h) isoform hCA VII, was involved in the oxidative stress defence processes, interfering with the generation of reactive oxygen species (ROS). ${ }^{16-17}$ Furthermore, isoform hCA IX, which is overexpressed in a wide range of hypoxic tumors, where it participates to the survival, proliferation, and metastasis processes, was recently investigated by our groups for its interaction with selenium-containing CA inhibitors (CAIs).$^{18}$ For these reasons we decided to also investigate various $\mathrm{Te}(\mathrm{II})$ derivatives as human (h) CA inhibitors (CAIs).

\section{Results and discussion}

\subsection{Chemistry}

Our long dated interest in the study of chalcogen-containing compounds ${ }^{22}$ led us to disclose synthetic routes towards organoselenides ${ }^{23}$ and, more recently, organotellurides ${ }^{24}$ through the ring opening of 
strained heterocycles with selenium and tellurium nucleophilic species. Some of these novel organic chalcogenides exhibited interesting antioxidant properties. ${ }^{25} \mathrm{With}$ the aim to evaluate the CA inhibitor activity of a series of Te-containing compounds, a wide variety of functionalized dialkyl and aryl-alkyl tellurides has thus been obtained following the Scheme 1. Dialkyl substituted $\beta$-hydroxy- and $\beta$-aminotellurides $\mathbf{4}$ and $\mathbf{5}$ were synthesised by treating $\mathrm{Li}_{2} \mathrm{Te}$ with epoxides $\mathbf{1}$ and aziridines $\mathbf{3}$, respectively. Through the same route, dithiatellurepane 6 was achieved from thiirane 2. $\beta$-Phenyltelluro thiol 7 and the corresponding disulfide $\mathbf{8}$ were selectively obtained from thiirane $\mathbf{2}$ and diphenyl ditelluride. Reaction of $\mathrm{PhTe}^{-}$, generated through reduction of $\mathrm{PhTeTePh}$, with epoxides and aziridines led smoothly to the formation of alcohols 9 and amines $\mathbf{1 0 .}$

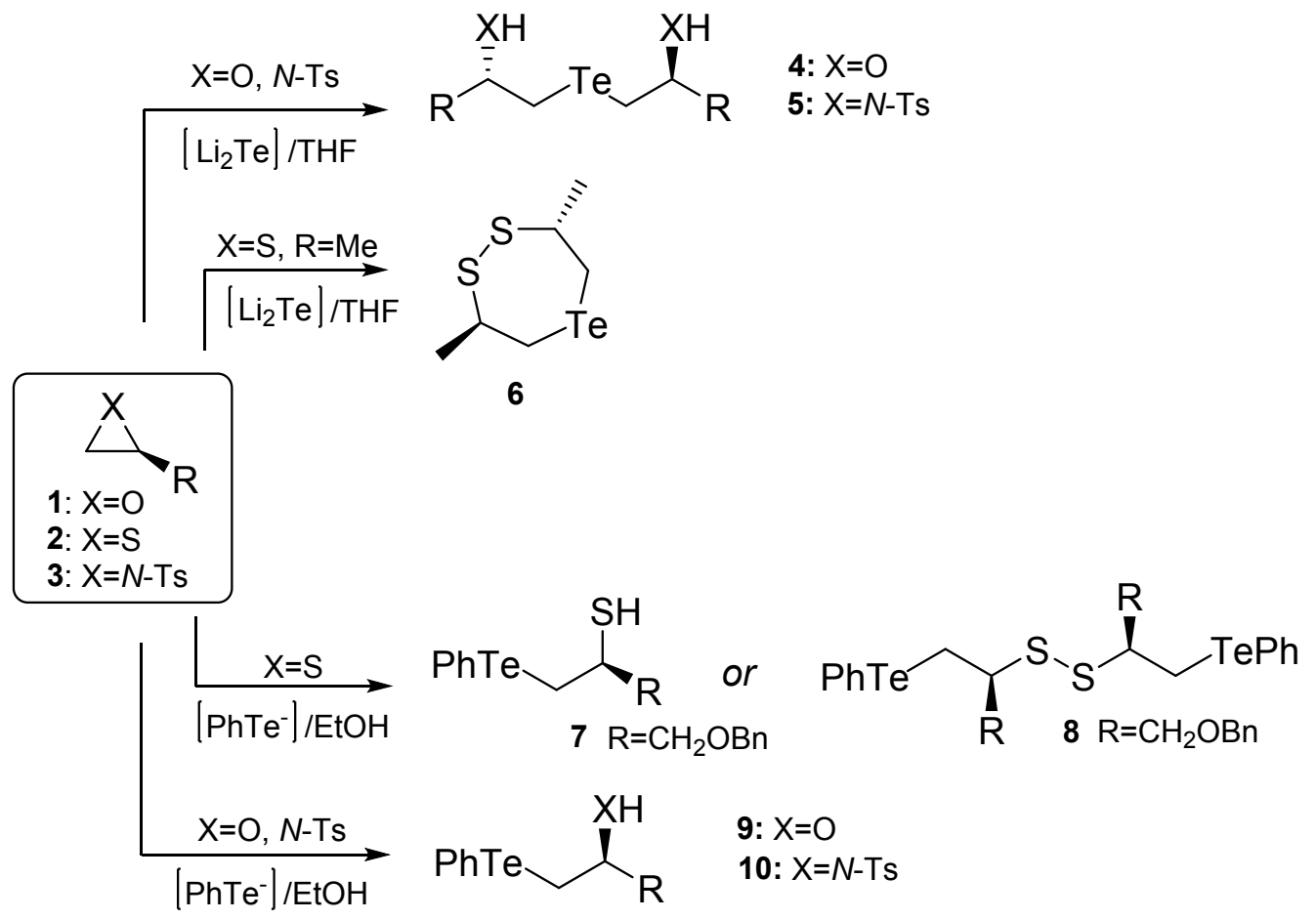

Scheme 1: Synthesis of $\beta$-functionalized tellurides. $\mathrm{Li}_{2} \mathrm{Te}$ is generated in situ from elemental $\mathrm{Te}(0.5$ eq. $)$ and $\mathrm{LiEt}_{3} \mathrm{BH}$ (1.0 eq.). $\mathrm{PhTe}^{-}$is generated in situ from $\mathrm{PhTeTePh}$ (0.5 eq.) and $\mathrm{NaBH}_{4}$ (1.5 eq.). 
Following a similar synthetic approach, novel chiral $N$-Tosyl $\beta$-aryltelluro amines were synthesised from diaryl ditellurides and enantioenriched aziridines, obtained from natural aminoacids, through a high regioselective and enantiospecific ring opening route (Scheme 2).

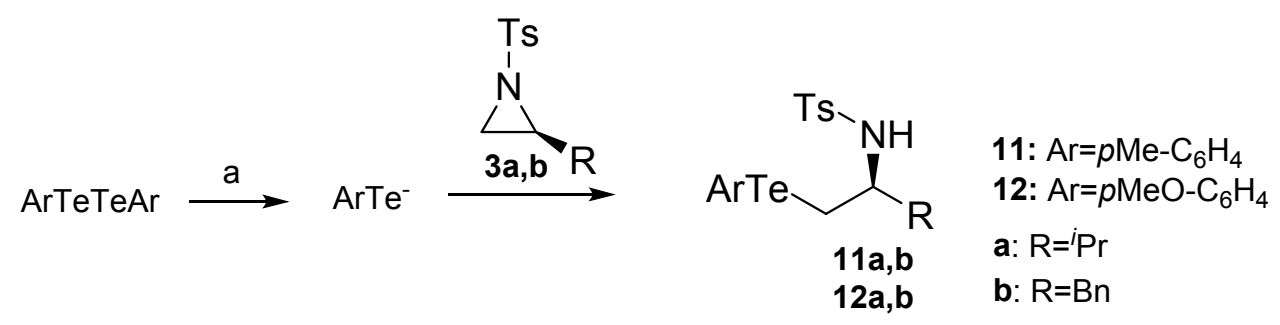

Scheme 2: Synthesis of $\beta$-aryltelluro amines. Reagent and conditions: a) $\operatorname{ArTeTe} A r(0.5$ eq. $), \mathrm{NaBH}_{4}(1.5$ eq.), $\mathrm{EtOH}, 0^{\circ} \mathrm{C}$.

The synthesised Te-containing CA inhibitors candidates are reported in Chart 1.<smiles>[R][C](O)C[Te]CC([R])O</smiles>

a: $\mathrm{R}=\mathrm{CH}_{2} \mathrm{OBn}$

b: $\mathrm{R}=\mathrm{Me}$

c: $\mathrm{R}=\mathrm{CH}_{2} \mathrm{OAll}$

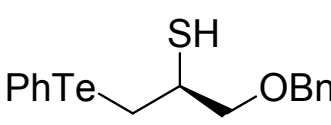

7<smiles>[R]C(C[PbH])N[AsH]</smiles>

10

a: $\mathrm{R}=(S)^{-}{ }^{-} \mathrm{Pr}$

b: $\mathrm{R}=(S)-\mathrm{Bn}$

c: $\mathrm{R}=(S)-\mathrm{Me}$ 。<smiles>[R]C(C[Te]CC([R])N[3H])N[3H]</smiles>

a: $R=(S)-i P r$

b: $\mathrm{R}=(S)-\mathrm{Me}$<smiles>c1ccc(COC[C@H](C[Te]c2ccccc2)SS[C@H](COCc2ccccc2)C[Te]c2ccccc2)cc1</smiles><smiles>C[C@H]1C[Te]C[C@H](C)SS1</smiles>

6<smiles>[R]C(O)C[PbH]</smiles>

9

a: $\mathrm{R}=\mathrm{CH}_{2} \mathrm{OBn}$

b: $\mathrm{R}=\mathrm{Me}$

Chart 1: Tellurium-containing chemotypes investigated as CA inhibitors in this work 


\subsection{Carbonic anhydrase inhibition}

All compounds $\mathbf{4 a - 1 2 b}$ were tested in vitro for their inhibitory activity against the physiologically relevant hCA isoforms I, II, IV, VII and IX by means of the stopped-flow carbon dioxide hydration assay ${ }^{26}$ and their activities were compared to the standard CAI acetazolamide (AAZ) (Table 1).

Table 1. Inhibition data of human CA isoforms I, II, IV, VII and IX with compounds $\mathbf{4 a - 1 2 b}$ and AAZ by a stopped flow $\mathrm{CO}_{2}$ hydrase assay. ${ }^{26}$

\begin{tabular}{llllll}
\hline Cmp & hCA I & hCA II & $\begin{array}{l}\text { K }(\mu \mathrm{M})^{*} \\
\text { hCA IV }\end{array}$ & hCA VII & hCA IX \\
\hline $\mathbf{4 a}$ & $>100$ & $>100$ & $>100$ & $>100$ & $>100$ \\
$\mathbf{4 b}$ & $>100$ & $>100$ & $>100$ & $>100$ & $>100$ \\
$\mathbf{4 c}$ & $>100$ & $>100$ & $>100$ & $>100$ & $>100$ \\
$\mathbf{5 a}$ & 0.71 & 0.03 & 60.8 & 0.62 & 3.4 \\
$\mathbf{5 b}$ & 18.0 & 0.09 & $>100$ & 5.3 & 19.7 \\
$\mathbf{6}$ & $>100$ & $>100$ & $>100$ & $>100$ & $>100$ \\
$\mathbf{7}$ & $>100$ & $>100$ & $>100$ & $>100$ & $>100$ \\
$\mathbf{8}$ & $>100$ & $>100$ & $>100$ & $>100$ & $>100$ \\
$\mathbf{9 a}$ & $>100$ & $>100$ & $>100$ & $>100$ & $>100$ \\
$\mathbf{9 b}$ & $>100$ & $>100$ & $>100$ & $>100$ & $>100$ \\
$\mathbf{1 0 a}$ & 27.3 & 35.8 & 85.8 & 3.2 & 1.5 \\
$\mathbf{1 0 b}$ & $>100$ & 56.8 & $>100$ & 4.8 & 11.0 \\
$\mathbf{1 0 c}$ & 76.5 & 61.6 & $>100$ & 0.05 & $>100$ \\
$\mathbf{1 1 a}$ & $>100$ & 41.6 & $>100$ & 5.0 & 0.7 \\
$\mathbf{1 1 b}$ & $>100$ & 31.4 & $>100$ & 3.2 & 2.0 \\
$\mathbf{1 2 a}$ & $>100$ & 56.3 & $>100$ & 4.1 & 2.3 \\
$\mathbf{1 2 b}$ & $>100$ & 56.1 & $>100$ & 4.9 & 11.7 \\
$\mathbf{A A Z}$ & 0.25 & 0.012 & 0.074 & 0.006 & 0.025 \\
\hline
\end{tabular}

* Mean from 3 different assays, by a stopped flow technique (errors were in the range of $\pm 5-10 \%$ of the reported values).

We have investigated a range of telluride and ditelluride derivatives for their interaction with the five hCA here considered, after a period of $15 \mathrm{~min}$ of incubation of the enzyme and inhibitor solutions ${ }^{26-28}$. The following structure activity relationship (SAR) may be noted regarding the inhibition data of Table 1: 
Cytosolic isoforms (hCA I, hCA II and hCA VII) were inhibited by isopropyl substituted amino telluride 5a in nanomolar range with particulary efficacy against hCA II $\left(\mathrm{K}_{\mathrm{i}} 0.03 \mu \mathrm{M}\right)$. Indeed, membrane isoforms here considered was inhibited by 5a in low micromolar range for transmembrane hCA IX $\left(\mathrm{K}_{\mathrm{i}} 3.4 \mu \mathrm{M}\right)$ and medium micromolar range for hCA IV $\left(\mathrm{K}_{\mathrm{i}} 60.4 \mu \mathrm{M}\right)$. The inhibition potency decreased when isopropyl moiety was replaced with methyl group (5b). On the other hand, the isopropyl moiety increased the selectivity versus hCA II with an activity 200 folds greater than against hCA I and nearly 60 times greater thans against hCA VII. Furthermore, this substitution caused a loss of activity for membrane isoform hCA IV. Dithiatellurepane 6, $\beta$ hydroxy tellurides (4a-c), $\beta$ phenyltelluro substituted thiol 7 , disulfide 8 and alcohols 9a-b did not inhibit the human isoforms here considered. $\beta$ phenyl telluro amine 10a showed a good selectivity for hCA IX. This compound was 20 times more potent than two dominant cytosolic isoforms (hCA I hCA II) and 2 folds more active compared to hCA VII. An interesting inhibition profile was observed for compounds 11a and 12a, where the introduction of para substituents in the aryl telluro scaffolds led to a loss of activity for hCA I and hCA IV. Moreover, for compound 11a, $\mathrm{CH}_{3}$ group in position 4 of the aryl telluride, increased 7 times the selectivity for hCA IX compared to hCA VII. The benzyl moiety in compounds $\mathbf{1 0 b}$ and $\mathbf{1 2 b}$ moved the selectivity for hCA VII and caused a loss of inhibitory power against hCA I and hCA IV. Another interesting point, was the inhibitory activity of 10c. Methyl moiety increased over 1000 folds the selectivity for hCA VII among the other cytosolic isoforms ( hCA I and hCA II), and led to loss the activity for the membrane isoforms hCA IV and hCA IX.

\section{Conclusions}


We have investigated a serie of tellurides as inhibitors on five $\alpha$-carbonic anhydrases (CAs, EC 4.2.1.1) of pharmacologic relevance, i.e., hCA I, II, IV, VII and IX. These isoforms are drug targets for antiglaucoma (hCA I, II and IV), antiepileptic (hCA VII) or antitumor (hCA IX) agents. In this contest, the investigated organotellurium compounds showed inhibitory action and in same case selectivity against the cytosolic over membrane-bound isoforms for compounds $\mathbf{5 a - b}$ and $\mathbf{1 0 c}$. On the other hand, compounds 10a, 11a-b and 12a showed selectivity against the membrane tumor-associated hCA IX over cytosolic isoforms. In the future, it will be interesting to test organotellurium compounds as antiinfectives against key enzymes that are expressed by pathogenic bacteria, such as the $\beta$ - and $\gamma$-carbonic anhydrases from Burkholderia pseudomallei ${ }^{29}$ and Francisella tularensis ${ }^{30}$ the etiological agents of meliodosis and tularemia, respectively.

\section{Experimental Part}

\subsection{General}

All reactions were carried out in an oven-dried glassware under inert atmosphere $\left(\mathrm{N}_{2}\right)$. All commercial materials were used as received without further purification. Flash column chromatography purifications were performed on Silica gel 60 (230-400 mesh). Thin layer chromatography was performed on TLC plates Silica gel 60 F254. NMR spectra were recorded in $\mathrm{CDCl}_{3}$ with Varian Gemini 200, Mercury 400 , and Bruker 400 Ultrashield spectrometers operating at 200 and $400 \mathrm{MHz}$ (for ${ }^{1} \mathrm{H}$ ), 50 and $100 \mathrm{MHz}$ (for ${ }^{13} \mathrm{C}$ ), and $126 \mathrm{MHz}$ (for ${ }^{125} \mathrm{Te}$ ). NMR signals were referenced to nondeuterated residual solvent signals (7.26 ppm for $1 \mathrm{H}, 77.0 \mathrm{ppm}$ for $13 \mathrm{C})$. (PhTe $)_{2}$ was used as an external reference for ${ }^{125} \mathrm{Te} \mathrm{NMR}(\delta=420$ ppm). ${ }^{1} \mathrm{H}$ NMR data are reported as follows: chemical shift, integration, multiplicity $(\mathrm{s}=$ singlet, $\mathrm{d}=$ doublet, ap $\mathrm{d}=$ apparent doublet, $\mathrm{m}=$ multiplet, $\mathrm{dd}=$ doublet of doublet, ecc.), coupling constant $(J)$ or line separation (ls), and assignment. 


\subsubsection{General procedure for the synthesis of $\beta$-hydroxy tellurides (4), $\beta$-amino tellurides (5) and} dithiatellurepane (6).

A suspension of $\mathrm{Li}_{2} \mathrm{Te}$ in $\mathrm{THF}$, generated from elemental tellurium (0.5 mmol, 1.0 eq.) and $\mathrm{LiEt}_{3} \mathrm{BH}$ (1.0mmol, 2.0 eq.), was treated with the electrophile (epoxide, aziridine or thiirane - $1.0 \mathrm{mmol}, 2.0$ eq.) and the reaction was stirred for $12 \mathrm{~h}$ at ambient temperature. Afterwards, the mixture was diluted with $\mathrm{Et}_{2} \mathrm{O}(10 \mathrm{~mL})$, filtered through a short plug of celite, washed with $\mathrm{NH}_{4} \mathrm{Cl}$ and then with $\mathrm{H}_{2} \mathrm{O}(2 \times 5 \mathrm{~mL})$. The organic phase was dried over $\mathrm{Na}_{2} \mathrm{SO}_{4}$, filtered and evaporated under reduced pressure. The crude residue was then purified by flash chromatography to yield $\beta$-functionalised tellurides.

\subsubsection{Procedure for the synthesis of $\beta$-phenyltelluro thiol (7)}

$\mathrm{NaBH}_{4}(28 \mathrm{mg}, 0.75 \mathrm{mmol}, 3.0$ eq.) was portionwise added to a solution of diphenyl ditelluride (102 mg, $0.25 \mathrm{mmol}, 1.0$ eq. $)$ in $\mathrm{EtOH}(2 \mathrm{~mL})$ at $0{ }^{\circ} \mathrm{C}$ under inert atmosphere $\left(\mathrm{N}_{2}\right)$. After $30 \mathrm{~min}$, the thiirane $2(0.5$ mmol, 2.0 eq.) was slowly added and the reaction mixture was stirred at $0{ }^{\circ} \mathrm{C}$ and the reaction progress was monitored by TLC. When the starting thiirane had completely reacted (monitored by TLC), $2 \mathrm{~mL}$ of a $50 \%$ aqueous solution of citric acid were added and the organic phase was extracted with $\mathrm{Et}_{2} \mathrm{O}(2 \times 5$ $\mathrm{mL})$, washed with brine $(1 \times 5 \mathrm{~mL})$, dried over $\mathrm{Na}_{2} \mathrm{SO}_{4}$, filtered and concentrated under vacuum. The crude material was purified by flash chromatography to yield $\beta$-phenyltelluro thiol 7.

\subsubsection{Procedure for the synthesis of $\beta$-phenyltelluro disulfide (8)}

$\mathrm{PhTe}^{-}$, generated as described for the synthesis of thiol 7, was reacted with thiirane 2 at $0{ }^{\circ} \mathrm{C}$ and the reaction mixture was allowed to warm to ambient temperature before leaving to react $14 \mathrm{~h}$ at the same temperature. Then, $2 \mathrm{~mL}$ of $\mathrm{H}_{2} \mathrm{O}$ were added and the organic phase was extracted with $\mathrm{Et}_{2} \mathrm{O}(2 \times 5 \mathrm{~mL})$, washed with brine $(1 \times 5 \mathrm{~mL})$, dried over $\mathrm{Na}_{2} \mathrm{SO}_{4}$, filtered and concentrated under reduced pressure. The crude material was purified by flash chromatography to yield $\beta$-phenyltellurodisulfides 8 . 


\subsubsection{General Procedure for the synthesis of $\beta$-phenyltelluro alcohols (9) and $N$-Ts protected $\beta$ -} aryltelluro amines (10-12).

$\mathrm{NaBH}_{4}$ (28 mg, $0.75 \mathrm{mmol}, 3.0$ eq.) was portionwise added to a solution of diaryl ditelluride ( $0.25 \mathrm{mmol}$, 1.0 eq.) in $\mathrm{EtOH}(2 \mathrm{~mL})$ at $0^{\circ} \mathrm{C}$ under inert atmosphere $\left(\mathrm{N}_{2}\right)$. After $30 \mathrm{~min}$, the electrophile (epoxide or aziridine $-0.45 \mathrm{mmol}, 1.8 \mathrm{eq}$.) was slowly added and the reaction mixture was stirred at room temperature until complete consumption of the starting material was observed by TLC. The reaction was quenched by addition of saturated aq. $\mathrm{NH}_{4} \mathrm{Cl}(2 \mathrm{~mL})$ and diluted with $\mathrm{Et}_{2} \mathrm{O}(5 \mathrm{~mL})$. The layers were separated and the organic layer was washed with $\mathrm{H}_{2} \mathrm{O}(3 \mathrm{~mL})$, dried over $\mathrm{Na}_{2} \mathrm{SO}_{4}$, filtered and concentrated under vacuum. The crude material was purified by flash chromatography to yield $\beta$-phenyltelluro alcohols (9) or $\beta$ aryltelluro amines (10-12).

\subsection{5 (S)-4-methyl- $N$-(3-methyl-1-(phenyltellanyl)butan-2-yl)benzenesulfonamide (10a)}

Following the general procedure, diphenyl ditelluride (51 mg, $0.125 \mathrm{mmol})$ and $(S)$-2-isopropyl-1tosylaziridine $(54 \mathrm{mg}, 0.23 \mathrm{mmol})$ gave after flash chromatography (petroleum ether/Et $\left.{ }_{2} \mathrm{O} 7: 1\right) \mathbf{1 0 a}(80$ mg, 78\%). ${ }^{1} \mathbf{H}$ NMR (400 MHz, $\left.\mathrm{CDCl}_{3}\right) \delta(\mathrm{ppm}): 0.74(3 \mathrm{H}, \mathrm{d}, J=6.7 \mathrm{~Hz}), 0.80(3 \mathrm{H}, \mathrm{d}, J=6.8 \mathrm{~Hz}), 1.78-$ $1.90(1 \mathrm{H}, \mathrm{m}), 2.38\left(3 \mathrm{H}, \mathrm{s}, \mathrm{CH}_{3}\right), 2.72\left(1 \mathrm{H}, \mathrm{dd}, J=6.5,12.1 \mathrm{~Hz}, \mathrm{CH}_{\mathbf{a}} \mathrm{H}_{\mathrm{b}} \mathrm{Te}\right), 3.10(1 \mathrm{H}, \mathrm{dd}, J=4.6,12.1 \mathrm{~Hz}$, $\left.\mathrm{CH}_{\mathrm{a}} \mathbf{H}_{\mathbf{b}} \mathrm{Te}\right), 3.18-3.20(1 \mathrm{H}, \mathrm{m}, \mathrm{CHNH}), 4.75(1 \mathrm{H}, \mathrm{d}, J=8.8 \mathrm{~Hz}, \mathrm{NH}), 7.17-7.20(4 \mathrm{H}, \mathrm{m})$, 7.29-7.33 (1H, m), 7.61-7.63 (4H, m). ${ }^{13} \mathbf{C}$ NMR (100 MHz, $\left.\mathrm{CDCl}_{3}\right) \delta(\mathrm{ppm}): 14.6\left(\mathrm{CH}_{2} \mathrm{Te}\right), 17.5,19.2,32.3,59.3,110.9$ (TeC), 127.0, 128.0, 129.3, 129.6, 137.8, 138.8, 143.2. MS (ESI positive) $m / z(\%): 468[\mathrm{M}+\mathrm{Na}]^{+},(100)$. Elemental analysis: $\mathrm{C}_{18} \mathrm{H}_{23} \mathrm{NO}_{2} \mathrm{STe}$ Calcd. C 48.58\%, H 5.21\%, N 3.15\%. Found: C 48.49\%, H 5.23\%, N $3.17 \%$.

\subsection{6 (S)-4-methyl- $N$-(1-phenyl-3-(phenyltellanyl)propan-2-yl)benzenesulfonamide (10b)}


Following the general procedure, diphenyl ditelluride (51 $\mathrm{mg}, 0.125 \mathrm{mmol})$ and $(S)$-2-benzyl-1tosylaziridine $(65 \mathrm{mg}, 0.23 \mathrm{mmol})$ gave after flash chromatography (petroleum ether/Et $\left.{ }_{2} \mathrm{O} 3: 1\right) \mathbf{1 0 b}(105$ mg, 93\%). ${ }^{1} \mathbf{H}$ NMR (400 MHz, $\left.\mathrm{CDCl}_{3}\right) \delta(\mathrm{ppm}): 2.38\left(3 \mathrm{H}, \mathrm{s}, \mathrm{CH}_{3}\right), 2.74(1 \mathrm{H}, \mathrm{dd}, J=6.8,13.8 \mathrm{~Hz}), 2.84-$ $2.89(2 \mathrm{H}, \mathrm{m}), 3.12(1 \mathrm{H}, \mathrm{dd}, J=4.3,12.4 \mathrm{~Hz}), 3.51-3.59(1 \mathrm{H}, \mathrm{m}, \mathrm{CHNH}), 4.78(1 \mathrm{H}, \mathrm{d}, J=7.5 \mathrm{~Hz}, \mathrm{NH}), 6.90-$ $6.92(2 \mathrm{H}, \mathrm{m}), 7.10-7.22(7 \mathrm{H}, \mathrm{m}), 7.29-7.33(1 \mathrm{H}, \mathrm{m}), 7.45(2 \mathrm{H}$, ap d, $1 \mathrm{~s}=8.3 \mathrm{~Hz}), 7.65-7.67(2 \mathrm{H}, \mathrm{m}) .{ }^{13} \mathrm{C}$ NMR $\left(100 \mathrm{MHz}, \mathrm{CDCl}_{3}\right) \delta(\mathrm{ppm}): 16.1\left(\mathrm{CH}_{2} \mathrm{Te}\right), 21.5,42.0,55.3,111.1(\mathrm{TeC}), 126.7,127.0,128.0,128.6$, 129.2, 129.4, 129.5, 136.5, 137.0, 138.6, 143.1. ${ }^{125}$ Te NMR (126 MHz, $\left.\mathrm{CDCl}_{3}\right) \delta(\mathrm{ppm}): 386.4$. MS (ESI positive) $m / z$ (\%): $516[\mathrm{M}+\mathrm{Na}]^{+}$, (100). Elemental analysis: $\mathrm{C}_{23} \mathrm{H}_{23} \mathrm{NO}_{2} \mathrm{STe}$ Calcd. C 53.59\%, $\mathrm{H} 4.70 \%$, N $2.84 \%$. Found: C $53.47 \%$, H $4.73 \%$, N $2.86 \%$.

\subsection{7 (S)-4-methyl- $N$-(1-(phenyltellanyl)propan-2-yl)benzenesulfonamide (10c)}

Following the general procedure, diphenyl ditelluride $(51 \mathrm{mg}, 0.125 \mathrm{mmol})$ and $(S)$-2-methyl-1tosylaziridine $(47 \mathrm{mg}, 0.23 \mathrm{mmol})$ gave after flash chromatography (petroleum ether/Et $2 \mathrm{O} \mathrm{2:1)} \mathrm{10c} \mathrm{(78}$ mg, 81\%). ${ }^{1} \mathbf{H}$ NMR $\left(400 \mathrm{MHz}, \mathrm{CDCl}_{3}\right) \delta(\mathrm{ppm}): 1.16(3 \mathrm{H}, \mathrm{d}, J=6.5 \mathrm{~Hz}), 2.43\left(3 \mathrm{H}, \mathrm{s}, \mathrm{CH}_{3}\right), 2.87(1 \mathrm{H}, \mathrm{dd}$, $\left.J=6.6,12.4 \mathrm{~Hz}, \mathrm{CH}_{\mathbf{a}} \mathrm{H}_{\mathrm{b}} \mathrm{Te}\right), 3.06\left(1 \mathrm{H}, \mathrm{dd}, J=4.7,12.4 \mathrm{~Hz}, \mathrm{CH}_{\mathrm{a}} \mathbf{H}_{\mathbf{b}} \mathrm{Te}\right), 3.48-3.71(1 \mathrm{H}, \mathrm{m}, \mathrm{CHNH}), 4.69$ $(1 \mathrm{H}, \mathrm{d}, J=7.7 \mathrm{~Hz}, \mathrm{NH}), 7.18-7.34(5 \mathrm{H}, \mathrm{m}), 7.64-7.69(4 \mathrm{H}, \mathrm{m}) .{ }^{13} \mathbf{C} \mathbf{N M R}\left(100 \mathrm{MHz}, \mathrm{CDCl}_{3}\right) \delta(\mathrm{ppm}): 18.6$ $\left(\mathrm{CH}_{2} \mathrm{Te}\right), 21.5,22.9,50.1,111.0(\mathrm{TeC}), 127.0,128.0,128.8,129.4,129.7,138.6,143.4$. MS (ESI positive) $m / z$ (\%): $440[\mathrm{M}+\mathrm{Na}]^{+},(100)$. Elemental analysis: $\mathrm{C}_{16} \mathrm{H}_{19} \mathrm{NO}_{2} \mathrm{STe}$ Calcd. C 46.09\%, H 4.59\%, N 3.36\%. Found: C 46.16\%, H 4.56\%, N 3.35\%.

\subsection{8 (S)-4-methyl- $N$-(3-methyl-1-(p-tolyltellanyl)butan-2-yl)benzenesulfonamide (11a)}

Following the general procedure, 1,2-di-p-tolylditellane $(55 \mathrm{mg}, 0.125 \mathrm{mmol}$ ) and $(S)$-2-isopropyl-1tosylaziridine (54 mg, $0.23 \mathrm{mmol}$ ) gave after flash chromatography (petroleum ether/EtOAc 5:1) $11 \mathrm{a}$ (76 mg, $72 \%) .{ }^{1} \mathbf{H}$ NMR $\left(400 \mathrm{MHz}, \mathrm{CDCl}_{3}\right) \delta(\mathrm{ppm}): 0.74\left(3 \mathrm{H}, \mathrm{d}, J=6.7 \mathrm{~Hz}, \mathrm{CH}_{3}\right), 0.8\left(3 \mathrm{H}, \mathrm{d}, J=6.8 \mathrm{~Hz}, \mathrm{CH}_{3}\right)$, 
1.8-1.9 (1H, m), $2.36\left(3 \mathrm{H}, \mathrm{s}, \mathrm{CH}_{3}\right), 2.39\left(3 \mathrm{H}, \mathrm{s}, \mathrm{CH}_{3}\right), 2.66\left(1 \mathrm{H}, \mathrm{dd}, J=6.6,12.2 \mathrm{~Hz}, \mathrm{CH}_{\mathbf{a}} \mathrm{H}_{\mathrm{b}} \mathrm{Te}\right), 3.05(1 \mathrm{H}$, dd, $\left.J=4.6,12.2 \mathrm{~Hz}, \mathrm{CH}_{\mathrm{a}} \mathbf{H}_{\mathbf{b}} \mathrm{Te}\right), 3.11-3.17(1 \mathrm{H}, \mathrm{m}, \mathrm{CHNH}), 4.75(1 \mathrm{H}, \mathrm{d}, J=8.9 \mathrm{~Hz}, \mathrm{NH}), 7.0$ (2H, ap d, $1 \mathrm{~s}=7.9 \mathrm{~Hz}), 7.17(2 \mathrm{H}$, ap d, $1 \mathrm{~s}=8 \mathrm{~Hz}), 7.52(2 \mathrm{H}$, ap d, $1 \mathrm{~s}=7.9 \mathrm{~Hz}), 7.62(2 \mathrm{H}$, ap d, $1 \mathrm{~s}=8 \mathrm{~Hz}) .{ }^{13} \mathbf{C ~ N M R}(100$ $\left.\mathrm{MHz}, \mathrm{CDCl}_{3}\right) \delta(\mathrm{ppm}): 14.5\left(\mathrm{CH}_{2} \mathrm{Te}\right), 17.5,19.2,21.2,21.5,32.3,59.3,106.7(\mathrm{TeC}), 127.0,129.5,130.2$ 137.8, 138.1, 139.2, 143.1. MS (ESI positive) $\mathrm{m} / z(\%): 482[\mathrm{M}+\mathrm{Na}]^{+},(100)$. Elemental analysis: $\mathrm{C}_{19} \mathrm{H}_{25} \mathrm{NO}_{2} \mathrm{STe}$ Calcd. C 49.71\%, H 5.49\%, N 3.05\%. Found: C 49.65\%, H 5.51\%, N 3.06\%.

\subsection{9 (S)-4-methyl- $N$-(1-phenyl-3-(p-tolyltellanyl)propan-2-yl)benzenesulfonamide (11b)}

Following the general procedure, 1,2-di-p-tolylditellane (55 $\mathrm{mg}, 0.125 \mathrm{mmol})$ and $(S)$-2-benzyl-1tosylaziridine (65 mg, $0.23 \mathrm{mmol}$ ) gave after flash chromatography (petroleum ether/EtOAc 5:1) 11b (101 mg, 87\%). ${ }^{1} \mathbf{H}$ NMR (400 MHz, $\left.\mathrm{CDCl}_{3}\right) \delta(\mathrm{ppm}): 2.36\left(3 \mathrm{H}, \mathrm{s}, \mathrm{CH}_{3}\right), 2.39\left(3 \mathrm{H}, \mathrm{s}, \mathrm{CH}_{3}\right), 2.74(1 \mathrm{H}, \mathrm{dd}, J=6.8$, $13.8 \mathrm{~Hz}), 2.79-2.88(3 \mathrm{H}, \mathrm{m}), 3.08(1 \mathrm{H}, \mathrm{dd}, J=4.3,12.4 \mathrm{~Hz}), 3.47-3.57(1 \mathrm{H}, \mathrm{m}, \mathrm{CHNH}), 4.72(1 \mathrm{H}, \mathrm{d}, J=7.5$ $\mathrm{Hz}, \mathrm{NH}), 6.90-6.92(2 \mathrm{H}, \mathrm{m}), 7.02(2 \mathrm{H}, \mathrm{d}, 1 \mathrm{~s}=8.0 \mathrm{~Hz}), 7.11(2 \mathrm{H}, \mathrm{d}, 1 \mathrm{~s}=8.3 \mathrm{~Hz}), 7.12-7.17(3 \mathrm{H}, \mathrm{m}), 7.44$ $(2 \mathrm{H}, \mathrm{d}, 1 \mathrm{~s}=8.3 \mathrm{~Hz}), 7.56(2 \mathrm{H}, \mathrm{d}, 1 \mathrm{~s}=8.0 \mathrm{~Hz}) .{ }^{13} \mathbf{C ~ N M R}\left(100 \mathrm{MHz}, \mathrm{CDCl}_{3}\right) \delta(\mathrm{ppm}): 16.2\left(\mathrm{CH}_{2} \mathrm{Te}\right), 21.2$, 21.5, 41.7, 55.3, 106.9 (TeC), 126.7, 127.0, 128.6, 129.2, 129.5, 130.3, 136.6, 137.0, 138.1, 139.0, 143.1. ${ }^{125}$ Te NMR (126 MHz, $\left.\mathrm{CDCl}_{3}\right) \delta(\mathrm{ppm}):$ 371.3. MS (ESI positive) $m / z(\%): 530[\mathrm{M}+\mathrm{Na}]^{+},(100)$. Elemental analysis: $\mathrm{C}_{23} \mathrm{H}_{25} \mathrm{NO}_{2} \mathrm{STe}$ Calcd. C 54.48\%, H 4.97\%, N 2.76\%. Found: C 54.52\%, H 4.95\%, N 2.75\%.

\subsubsection{0 (S)-N-(1-((4-methoxyphenyl)tellanyl)-3-methylbutan-2-yl)-4-methylbenzenesulfonamide} (12a)

Following the general procedure, 1,2-bis(4-methoxyphenyl)ditellane (59 $\mathrm{mg}, 0.125 \mathrm{mmol})$ and $(S)$-2isopropyl-1-tosylaziridine (54 mg, $0.23 \mathrm{mmol}$ ) gave after flash chromatography (petroleum ether/EtOAc 5:1) 12a (81 mg, 74\%). ${ }^{1} \mathbf{H}$ NMR $\left(400 \mathrm{MHz}, \mathrm{CDCl}_{3}\right) \delta(\mathrm{ppm}): 0.73(3 \mathrm{H}, \mathrm{d}, J=6.7 \mathrm{~Hz}), 0.79(3 \mathrm{H}, \mathrm{d}, J=6.8$ $\mathrm{Hz}), 1.81-1.89(1 \mathrm{H}, \mathrm{m}), 2.39\left(3 \mathrm{H}, \mathrm{s}, \mathrm{CH}_{3}\right), 2.63\left(1 \mathrm{H}, \mathrm{dd}, J=6.7,12.2 \mathrm{~Hz}, \mathrm{CH}_{\mathrm{a}} \mathrm{H}_{\mathrm{b}} \mathrm{Te}\right), 3.02(1 \mathrm{H}, \mathrm{dd}, J=4.6$, 
$\left.12.2 \mathrm{~Hz}, \mathrm{CH}_{\mathrm{a}} \mathbf{H}_{\mathbf{b}} \mathrm{Te}\right), 3.1-3.15(1 \mathrm{H}, \mathrm{m}, \mathrm{CHNH}), 3.81\left(3 \mathrm{H}, \mathrm{s}, \mathrm{OCH}_{3}\right), 4.72(1 \mathrm{H}, \mathrm{d}, J=8.9 \mathrm{~Hz}, \mathrm{NH}), 6.74(2 \mathrm{H}$, $\mathrm{d}, 1 \mathrm{~s}=8.7 \mathrm{~Hz}), 7.18(2 \mathrm{H}, \mathrm{d}, 1 \mathrm{~s}=8 \mathrm{~Hz}), 7.57(2 \mathrm{H}, \mathrm{d}, 1 \mathrm{~s}=8.7 \mathrm{~Hz}), 7.61(2 \mathrm{H}, \mathrm{d}, 1 \mathrm{~s}=8 \mathrm{~Hz}) .{ }^{13} \mathbf{C ~ N M R}(100 \mathrm{MHz}$, $\left.\mathrm{CDCl}_{3}\right) \delta(\mathrm{ppm}): 14.8\left(\mathrm{CH}_{2} \mathrm{Te}\right), 17.5,19.2,21.5,32.2,55.2,59.4,99.8(\mathrm{TeC}), 115.3,127.0,129.5,137.9$, 141.3, 149.1. ${ }^{125}$ Te NMR $\left(126 \mathrm{MHz}, \mathrm{CDCl}_{3}\right) \delta(\mathrm{ppm}): 363.27 . \mathrm{MS}$ (ESI positive) $\mathrm{m} / z(\%): 498[\mathrm{M}+\mathrm{Na}]^{+}$, (100). Elemental analysis: $\mathrm{C}_{19} \mathrm{H}_{25} \mathrm{NO}_{3} \mathrm{STe}$ Calcd. C 48.04\%, H 5.30\%, N 2.95\%. Found: C 48.09\%, H $5.28 \%, \mathrm{~N} 2.96 \%$.

\subsubsection{1 (S)-N-(1-((4-methoxyphenyl)tellanyl)-3-phenylpropan-2-yl)-4-methylbenzenesulfonamide} $12 \mathrm{~b}$

Following the general procedure, 1,2-bis(4-methoxyphenyl)ditellane (30 mg, $0.063 \mathrm{mmol})$ and $(S)-2-$ benzyl-1-tosylaziridine (32 $\mathrm{mg}, 0.113 \mathrm{mmol}$ ) gave after flash chromatography (petroleum ether/EtOAc 5:1) 12b (54 mg, 91\%). ${ }^{1} \mathbf{H}$ NMR (400 MHz, $\left.\mathrm{CDCl}_{3}\right) \delta(\mathrm{ppm}): 2.39\left(3 \mathrm{H}, \mathrm{s}, \mathrm{CH}_{3}\right) ; 2.74-2.76(2 \mathrm{H}, \mathrm{m}) ; 2.79$ $(1 \mathrm{H}, \mathrm{dd}, J=6.5,13.8 \mathrm{~Hz}) ; 3.05(1 \mathrm{H}, \mathrm{dd}, J=4.3,12.4 \mathrm{~Hz}) ; 3.47-3.55(1 \mathrm{H}, \mathrm{m}, \mathrm{CHNH}) ; 3.82\left(3 \mathrm{H}, \mathrm{s}, \mathrm{OCH}_{3}\right)$; $4.75(1 \mathrm{H}, \mathrm{d}, J=8 \mathrm{~Hz}, \mathrm{NH}) ; 6.76(2 \mathrm{H}$, ap.d, ls=8.8 Hz); 6.79-6.91 (2H, m); 7.11-7.17 (5H, m); 7.43 (2H, d, ls $=8.3 \mathrm{~Hz}) ; 7.62\left(2 \mathrm{H}\right.$, ap.d, ls=8.8 Hz). ${ }^{13} \mathbf{C}$ NMR $\left(100 \mathrm{MHz}, \mathrm{CDCl}_{3}\right) \delta(\mathrm{ppm}): 16.4\left(\mathrm{CH}_{2} \mathrm{Te}\right) ; 21.5 ; 41.8$; $55.18 ; 55.23 ; 100.0(\mathrm{TeC}) ; 115.4 ; 126.7 ; 127.0 ; 128.6 ; 129.2 ; 129.5 ; 136.6 ; 137.0 ; 141.1 ; 143.1 .{ }^{125} \mathrm{Te}$ NMR (126 MHz, $\left.\mathrm{CDCl}_{3}\right) \delta(\mathrm{ppm}): 369.51 . \mathrm{MS}$ (ESI positive) $m / z(\%): 546[\mathrm{M}+\mathrm{Na}]^{+},(100)$. Elemental analysis: $\mathrm{C}_{23} \mathrm{H}_{25} \mathrm{NO}_{3} \mathrm{STe}$ Calcd. C 52.81\%, H 4.82\%, N 2.68\%. Found: C 52.75\%, H 4.84\%, N 2.69\%.

\subsection{Carbonic anhydrase inhibition}

An Applied Photophysics stopped-flow instrument has been used for assaying the $\mathrm{CA}$ catalyzed $\mathrm{CO}_{2}$ hydration activity. ${ }^{26}$ Phenol red (at a concentration of $0.2 \mathrm{mM}$ ) has been used as indicator, working at the absorbance maximum of $557 \mathrm{~nm}$, with 20 mMHepes (pH 7.5) as buffer, and $20 \mathrm{mM} \mathrm{Na}_{2} \mathrm{SO}_{4}$ (for maintaining constant the ionic strength), following the initial rates of the $\mathrm{CA}$-catalyzed $\mathrm{CO}_{2}$ hydration 
reaction for a period of $10-100 \mathrm{~s}$. The $\mathrm{CO}_{2}$ concentrations ranged from 1.7 to $17 \mathrm{mM}$ for the determination of the kinetic parameters and inhibition constants. For each inhibitor at least six traces of the initial 5$10 \%$ of the reaction have been used for determining the initial velocity. The uncatalyzed rates were determined in the same manner and subtracted from the total observed rates. Stock solutions of inhibitor $(0.1 \mathrm{mM})$ were prepared in distilled-deionized water and dilutions up to $0.01 \mathrm{nM}$ were done thereafter with the assay buffer. Inhibitor and enzyme solutions were preincubated together for 15 min at room temperature prior to assay, in order to allow for the formation of the E-I complex. The inhibition constants were obtained by non-linear least-squares methods using PRISM 3 and the Cheng-Prusoff equation, as reported earlier, ${ }^{27,28}$ and represent the mean from at least three different determinations. All CA isofoms were recombinant ones obtained in-house as reported earlier. ${ }^{27,28}$

\section{References}

1) Řezanka T., Sigler K., Phytochemistry, 2008, 69: 585.

2) Deng Z. T., Zhang Y., Yue J. C., Tang F. Q., Wei Q., J. Phys. Chem. B, 2007, 111: 12024.

3) Chen H., Lesnyak V., Bigall N. C., Gaponik N., Eychmüller A., Chem Mater, 2010, 22: 2309.

4) Wachter J., Eur. J. Inorg. Chem., 2004, 1367.

5) Zhang B., Hou W. Y., Ye X. C., Fu S. Q., Xie Y., Adv. Funct. Mater., 2007, 17: 486.

6) Zhang L., Wang C., Wen D. Y, Eur. J. Inorg. Chem., 2009, 3291.

7) Turner R. J., Weiner J. H., Taylor D. E., Microbiology, 1999, 145: 2549.

8) Fleming A., J. Pathol. Bacteriol., 1932, 35: 831.

9) Sredni B., Caspi R. R., Klein A., Kalechman Y., Danziger Y., BenYa'akov M., Tamari T., Shalit F., Albeck M., Nature, 1987, 330: 173.

10) Montero R., Gonsebatt M. E., Gerson R., Rojas E., Herrera L. A., Ostrosky-Wegman P., Anticancer Drugs, 1993, 3: 351. 
11) Sredni B., Caspi R. R., Lustig S., Klein A., Kalechman Y., Danziger Y., Ben Ya'akov M., Tamari T., Shalit F., Albeck M., Nat Immun Cell Growth Regul., 1988,7: 163.

12) Cunha R. L., Gouvea I. E., Juliano L., Acad Bras Cienc, 2009, 81: 393.

13) Giles G. I., Giles N. M., Collins C. A., Holt K., Fry F. H., Lowden P. A. S., Gutowski N. J., Jacob C., Chem. Commun., 2003, 2030.

14) Giles G. I., Tasker K. M., Johnson R. J. K., Jacob C., Peers C., Green K. N., Chem. Commun., $2001,2490$.

15). Monti D. M., De Simone G., Langella E., Supuran C. T., Di Fiore A., Monti S. M., J Enzyme Inhib Med Chem. 2016, 21: 1.

16) Cabiscol E., Levine R.L., J Biol Chem, 1995, 270: 14742.

17) Del Giudice R., Monti D.M., Truppo E., et al. Biol Chem, 2013, 394:1343.

18) (a) Swietach P., Vaughan-Jones R., Harris A., Cancer Metastasis Rev., 2007, 26: 299. (b) Supuran C.T., Metabolites. 2017, 7: E48. (c) Neri D., Supuran C.T., Nature Rev Drug Discov., 2011, 10: 767.

19) Supuran C.T., Nature Rev Drug Discov., 2008, 7: 168.

20) (a) Supuran C.T., J Enzyme Inhib Med Chem., 2012, 27: 759; (b) Supuran C.T, J Enzyme Inhib Med Chem., 2016, 31:345.

21) (a) Supuran C.T., Biochem J., 2016, 473: 2023; (b) Supuran C.T., Expert Opin Drug Discov., 2017, 12: 61; (c) Puccetti L., Fasolis G., Vullo D., Chohan Z.H., Scozzafava A., Supuran C.T. Bioorg Med Chem Lett. 2005, 15: 3096.

22) (a) Capperucci A., Tanini D., Phosphorus Sulfur Silicon Relat. Elem., 2015, 190: 1320. (b) Degl'Innocenti A., Pollicino S., Capperucci A., Chem. Commun., 2006, 4881.

23) (a) Tanini D., Capperucci A., Degl'Innocenti A., Eur. J. Org. Chem., 2015, 357. (b) Capperucci A., Tanini D., Borgogni C., Degl'Innocenti A., Heteroat. Chem., 2014, 25: 678. 
24) Tanini D., Grechi A., Dei S., Teodori E., Capperucci A., Tetrahedron, 2017, 73: 5646.

25) (a) Tanini D., Gori M., Bicocchi F., Ambrosi M., Lo Nostro P., Capperucci A., Arkivoc, 2017, part ii, 407. (b) Tanini D., D'Esopo V., Chen D., Barchielli G., Capperucci A., Phosphorus, Sulfur Silicon Relat. Elem., 2017, 192: 166. (c) Menichetti S., Capperucci A., Tanini D., Braga A.-L., Botteselle G.-V., Viglianisi C., Eur. J. Org. Chem., 2016, 3097. (d) Tanini D., Panzella L., Amorati R., Capperucci A., Pizzo E., Napolitano A., Menichetti S., D'Ischia M., Org. Biol. Chem., 2015, 13: 5757; (e) Scozzafava A., Briganti F., Mincione G., Menabuoni L., Mincione F., Supuran C.T., J Med Chem., 1999, 42: 3690.

26) Khalifah R. G., J. Biol. Chem., 1971, 246: 2561.

27) (a) Angeli A., Carta F., Bartolucci G., Supuran C.T., Bioorg Med Chem., 2017, 25: 3567. (b) Angeli A., Tanini D., Viglianisi C., Panzella L., Capperucci A., Menichetti S., Supuran C.T., Bioorg Med Chem., 2017, 25: 2518. (c) Mollica A., Locatelli M., Macedonio G., Carradori S., Sobolev A.P., De Salvador R.F., Monti S.M., Buonanno M., Zengin G., Angeli A., Supuran C.T., J. Enzyme Inhib. Med. Chem., 2016, 31 Sup 4: 1; (d) Supuran C.T., Nicolae A., Popescu A., Eur J Med Chem. 1996, 31: 431.

28) (a) De Vita D., Angeli A., Pandolfi F., Bortolami M., Costi R., Di Santo R., Suffredini E., Ceruso M., Del Prete S., Capasso C., Scipione L., Supuran C.T., J. Enzyme Inhib. Med. Chem., 2017, 32: 798. (b) Bruno E., Buemi M.R., Di Fiore A., De Luca L., Ferro S., Angeli A., Cirilli R., Sadutto D., Alterio V., Monti S.M., Supuran C.T., De Simone G., Gitto R., J Med Chem., 2017, 60: 4316. (c) Angeli A., Peat T.S., Bartolucci G., Nocentini A., Supuran C.T., Carta F., Org Biomol Chem., 2016, 48: 11353; (d) Supuran C.T., Barboiu M., Luca C, Pop E., Brewster M.E., Dinculescu A., Eur J Med Chem., 1996, 31: 597. 
29) (a) Del Prete S., Vullo D., Di Fonzo P., M. Osman S., AlOthman Z., Donald W.A., Supuran C.T., Capasso C., Bioorganic \& Medicinal Chemistry Letters, 2017, 27, 490. (b) Vullo D., Del Prete S., Di Fonzo P., Carginale V., Donald W.A., Supuran C.T., Capasso C., Molecules, 2017, 22, 421.

30) (a) Del Prete S. Vullo D., Osman S.M., AlOthman Z., Donald W.A., Winum J.Y., Supuran C.T., Capasso C., Bioorg Med Chem., 2017,25, 4800; (b) Capasso C., Supuran C.T., J Enzyme Inhib Med Chem. 2015, 30: 325, 